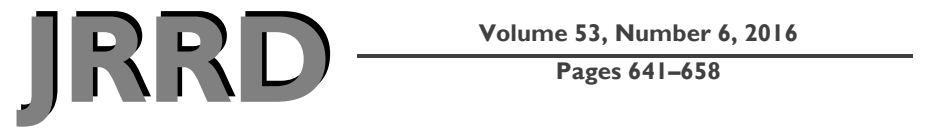

\section{Validity of activity monitors in wheelchair users: A systematic review}

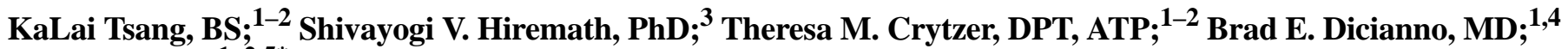 \\ Dan Ding, $\mathbf{P h D}^{1-2,5^{*}}$ \\ ${ }^{1}$ Human Engineering Research Laboratories, Department of Veterans Affairs (VA) Pittsburgh Healthcare System, \\ Pittsburgh, PA; ${ }^{2}$ Department of Rehabilitation Science and Technology, University of Pittsburgh, Pittsburgh, PA; \\ ${ }^{3}$ Department of Physical Therapy, Temple University, Philadelphia, PA; Departments of ${ }^{4}$ Physical Medicine and Reha- \\ bilitation and ${ }^{5}$ Bioengineering, University of Pittsburgh, Pittsburgh, PA
}

\begin{abstract}
Assessing physical activity (PA) in manual wheelchair users (MWUs) is challenging because of their different movement patterns in comparison to the ambulatory population. The aim of this review was to investigate the validity of portable monitors in quantifying PA in MWUs. A systematic literature search was performed. The data source was full reports of validation and evaluation studies in peer-reviewed journals and conference proceedings. Eligible articles between January 1, 1999, and September 18, 2015, were identified in three databases: PubMed, Institute of Electrical and Electronics Engineers, and Scopus. A total of 164 articles (158 from the databases and 6 from the citation/reference tracking) were identified, and 29 met the eligibility criteria. Two investigators independently extracted the characteristics from each selected article following a predetermined protocol and completed seven summary tables describing the study characteristics and key outcomes. In the identified studies, the monitors were used to assess three types of PA measures: energy cost, user movement, and wheelchair movement. The customized algorithms/ monitors did not estimate energy cost in MWUs as well as the commercial monitors did in the ambulatory population; however, they showed fair accuracy in measuring both wheelchair and user movements.
\end{abstract}

Key words: activities of daily living, activity tracking, energy expenditure, manual wheelchair users, motion sensors, physical activities, physical fitness, portable activity monitors, prediction models, wheelchair use.

\section{INTRODUCTION}

In the past decades, physical inactivity has become one of the biggest public health concerns in the United States and around the world [1]. According to a report from the Centers for Disease Control and Prevention, approximately 64 percent of the total healthcare expenditure in the United States is used to treat the chronic diseases that are associated with physical inactivity such as obesity, diabetes, cardiovascular diseases, and some cancers [2-3]. Although physical activity (PA) has numerous reported physical, physiological, and psychological benefits [4-8], many people with disabilities tend to lead a sedentary lifestyle, especially those who use wheelchairs as their primary means of mobility [9]. There are currently 3.3 million wheelchair users in the United States, and 1.5 million of them are manual wheelchair users

\footnotetext{
Abbreviations: $\mathrm{CI}$ = confidence interval, $\mathrm{EE}$ = energy expenditure, HR = heart rate, ICC = intraclass correlation coefficient, IEEE = Institute of Electrical and Electronics Engineers, MAE = mean absolute error, MSE = mean signed error, $\mathrm{MWU}=$ manual wheelchair user, $\mathrm{PA}=$ physical activity, RSI = repetitive strain injury, SCI = spinal cord injury.

*Address all correspondence to Dan Ding, PhD; Human Engineering Research Laboratories, 6425 Penn Ave, Suite 400, Pittsburgh, PA 15206; 412-822-3684. Email: dad5@pitt.edu http://dx.doi.org/10.1682/JRRD.2016.01.0006
} 
(MWUs) [10-11]. More than half (56\%) of adult wheelchair users, even if able, do not engage in regular PA at recommended levels [4,12-13]. Consequently, this population is three times more likely to have the aforementioned chronic diseases than people without disabilities [12-13]. Preventative measures such as promoting regular PA and an active lifestyle are some of the best ways to reduce the incidence of chronic illness and provide financial relief for an already strained medical system [14].

Quantifying PA is essential for determining the effectiveness of PA promotion programs and facilitating healthy behaviors and adherence to PA programs. It has been reported that people with disabilities are 82 percent more likely to be physically active if their healthcare providers recommend regular PA [13]. Clinicians, especially those who frequently interact with wheelchair users, play an important role in recognizing physical inactivity, motivating engagement in regular PA, and promoting health and wellness [15]. Baseline assessment of PA can help clinicians assist their clients with establishing PA participation goals. Other advantages include preventing physical inactivity, decreasing the severity of secondary conditions related to physical inactivity, limiting the degree of disability, and promoting regular PA $[9,16]$. Moreover, continuous and regular PA assessment allows clinicians to track the progress and compliance to the recommended interventions and assess the outcome of interventions [16]. Wheelchair users can also benefit from monitoring their own PA. Previous studies have shown that self-monitoring, along with feedback from experts, is a useful technique for increasing overall $\mathrm{PA}$, as it provides cues for people to enact desired behaviors [17-18].

Many methods exist for assessing PA, such as surveys, behavioral observations, physiological markers, calorimetry, and mechanical and electronic monitors [19]. Self-reported surveys and activity logs have been the most common approaches used by healthcare professionals to track their clients' PA. Although these methods are inexpensive and practical for large-scale populations, it is often burdensome for individuals to repeatedly record their PA throughout the day. In addition, the data collected rely on an individual's memory and societal desirability $[16,20]$, and thus, these self-reported tools may lack the accuracy and sensitivity needed to detect changes in PA on a daily basis [17-19,21]. Brown et al. reported inconsistent results obtained from four common PA measurement surveys that were used around the world [22].
With the advancement in microelectromechanical systems and wireless technologies, the use of portable PA monitors to objectively quantify daily activities has become popular in the general public. There are many commercially available monitors. The two most commonly used types are accelerometer- and multisensorbased monitors. Accelerometer-based monitors detect spatial changes in one, two, or three directions, while multisensor-based monitors detect spatial changes as well as physiological responses to bodily movement (e.g., heart rate [HR], near-body temperature, and skin conductance). The validity of these monitors has been widely investigated in ambulatory populations. Van Remoortel et al. conducted a systematic review on the validity of activity monitors in individuals with and without chronic diseases who were able to ambulate and showed that triaxial accelerometer and multisensor-based devices are valid in tracking PA, with mean percent differences of -6.85 percent (95\% confidence interval [CI]: $-18.20 \%$ to $4.49 \%$ ) and -3.64 percent (95\% CI: $-8.97 \%$ to $1.70 \%$ ), respectively, when compared with the gold standard (i.e., doubly labeled water) [21]. However, the monitors were evaluated only on activities that required unimpaired lower-limb function (i.e., walking, stair climbing, and running) [21].

Currently, no systematic review has been completed on the use of activity monitors in a nonambulatory population. Such a review is needed to (1) assist researchers to improve the quality of wearable health/fitness monitors for MWUs, (2) allow researchers and clinicians to assess the effectiveness of their PA interventions, and (3) allow clinicians to make recommendations about the use of technology in exercise and activity and monitor patients' compliance to interventions [23]. The purpose of this systematic review was to examine the use and validity of either commercially available monitors or custom monitors in quantifying PA-related outcomes in MWUs.

\section{METHODS}

\section{Inclusion/Exclusion Criteria}

Studies that met the following criteria were included in this review: (1) participants in the study had $\geq 1$ diagnosis resulting in a long-term functional or activity limitation that led to manual wheelchair use, (2) the instruments used in the study were commercial or custom-made products that were portable and designed for 
everyday use, and (3) the outcomes of the activity monitors were validated with gold standards or other validated tools. Review articles and studies that evaluated nonportable monitors or did not include MWUs in the validation protocol were excluded. No language restrictions were applied. Studies written in foreign languages were translated into English using a free online translator and checked by a native speaker to determine their eligibility.

\section{Data Sources and Literature Searches}

Three databases-PubMed, Institute of Electrical and Electronics Engineers (IEEE), and Scopus-were used for this review. A librarian was consulted to identify appropriate search terms for wheelchair users, activity monitors, and PA before initiating the search. For each of the databases, we searched the literature using the following key terms: wheelchair user, activity monitor, and physical activity. Synonyms and different spellings of each key term were determined and joined using "OR" (Appendix 1, available online only). The exploded results of the key terms were combined using "AND." Three filters were used to limit the search results. The article type (or document type) filter was used to elimi-nate review articles and notes. The publication dates filter was used to set publication date limits of January 1, 1999, to September 18, 2015. Articles published prior to 1999 were excluded because of the uncommon use of portable activity monitors for tracking PA before that time. The subject area (or publication title or journal categories) fil-ter was also used to remove articles in Biochemistry; Genetics and Molecular Biology; Physics and Astron-omy; Intelligent Robots and Systems; Rehabilitation Robotics; dental journals; Robotics and Automation Mag-azine: and Systems, Man and Cybernetics. The detailed search strategy used for PubMed is provided as an exam-ple in Appendix 1.

Corresponding authors of selected articles without full-text access were contacted for electronic copies of the articles. Full-text articles were downloaded, stored, and shared among investigators. Additional articles were identified through reference and citation tracking of the selected articles and relevant review articles.

\section{Study Selection Strategy}

The review team consisted of three members. Each member of the review team independently screened the titles and abstracts of the identified articles according to the inclusion/exclusion criteria. After the initial screen- ing, the assessments of each reviewer were compared. Differences in screening results were discussed among reviewers until consensus was reached. Articles that had all reviewers' consensus were downloaded for full-text evaluation. The same reviewers individually assessed the full text of the selected articles, and articles that had all reviewers' consensus were chosen for data analysis.

\section{Data Extraction}

The data extraction protocol was developed prior to the search. The studies were categorized into three groups: (1) studies that evaluated commercial monitors with default algorithms, (2) studies that evaluated commercial monitors with custom algorithms, and (3) studies that evaluated custom monitors that were primarily research prototypes. Seven tables were constructed that described the devices used and the details of the selected studies. Appendix 2 (available online only) is an overview of the commercial and custom devices used in the selected studies. Appendixes 3-5 (available online only) summarize the study design, while Tables 1-3 describe the key study results of every study in each of the three groups. After reviewing the full text of the articles, two investigators independently extracted the key characteristics from each selected article following the protocol and completed these tables. Results from each investigator were compared, and consensus was achieved upon discrepancies before compiling the results.

\section{RESULTS}

\section{Literature Searches}

The literature search from 3 databases yielded 158 unique citations. Based on title and abstract screening, 125 articles were rejected because they were review articles, did not include MWUs, did not evaluate or validate the devices by comparing to gold standard or reference comparisons, or evaluated devices that were not portable. Citation and reference tracking were performed on the remaining 33 articles that met the inclusion criteria, and as a result, 6 additional articles were identified for title and abstract screening. However, none of the 6 articles were included for full-text assessment because the inclusion criteria were not met. The same reviewers assessed the full text of the 33 articles ( 1 article required translation from Spanish to English), and 4 of them were rejected because they were conference abstracts, did not include 
JRRD, Volume 53, Number 6, 2016

Table 1.

Findings of six studies that evaluated commercial monitors with default algorithms.

\begin{tabular}{|c|c|c|c|}
\hline Study, Year & Monitor & Criterion Measures & Outcome Measures \\
\hline $\begin{array}{l}\text { Tanhoffer et al., } \\
2015 \text { [24] }\end{array}$ & SW & DLW & $\begin{array}{l}\text { Energy cost } \\
\text { TDEE: } R^{2}: 0.69, p=0.003 \\
\text { PAEE: } R^{2}: 0.16, p=0.13\end{array}$ \\
\hline $\begin{array}{l}\text { Hiremath et al., } \\
2012 \text { [29] }\end{array}$ & SW & Cosmed K4b2* & $\begin{array}{l}\text { Energy cost } \\
\text { MSE: }-55.3 \% \text { (95\% CI: }-62.5 \% \text { to }-48.1 \%) \\
\text { MAE: } 59.2 \%(95 \% \mathrm{CI}: 52.6 \% \text { to } 65.8 \%) \\
\text { BA: }-5.87,2.13 \mathrm{kcal} / \mathrm{min}\end{array}$ \\
\hline
\end{tabular}

Perez-Tejero et al., SW 2012 [25]

Hiremath et al., RT3, SW Cosmed K4b2* 2011 [27]

Hiremath et al., RT3 Cosmed K4b2* 2011 [28]

Warms and Belza, Actiwatch Self-reported PA record 2004 [26]

PASIPD questionnaire
Energy cost

Active EE (MET $>3): r=0.35, p<0.01$

MET: $r=0.52, p<0.01$

User movements

Duration of PA (i.e., time when MET $>3$ in $\geq 2$ consecutive min): $r=0.53, p<0.01$

\section{Energy cost}

RT3: MAE: $22.0 \%$ to 52.8\%; ICC(3,1): 0.64 (95\% CI: 0.51 to 0.73 ); Spearman rho: $0.72, p<0.05$

SW: MAE: $24.4 \%$ to $125.8 \%$; ICC(3,1): 0.62 (95\% CI: 0.49 to 0.72 ); Spearman rho: $0.84, p<0.05$

Energy cost

RT3 Waist: MAE: 21.3\% to 55.2\%

Range of MAE represented MAE's for different activities

\section{User movements}

All activities: $r=0.60$ (range: 0.30 to 0.77 )

Exclude time in vehicle: $r=0.59$ (range: 0.30 to 0.76 )

Exclude time in sleep: $r=0.40$ (range: 0.14 to 0.65 )

Note: An overview of the commercial and custom monitors can be found in Appendix 2 (available online only)

*COSMED, Rome, Italy.

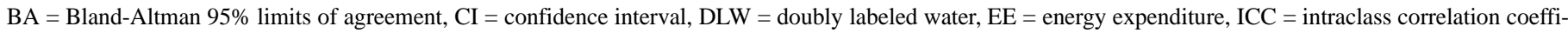

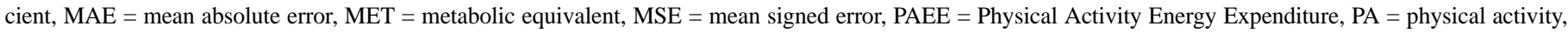
PASIPD = Physical Activity Scale for Individuals with Physical Disabilities, SW = SenseWear, TDEE = Total Daily Energy Expenditure.

MWUs, were not evaluation or validation studies, or did not evaluate monitors meant for everyday use. As a result, a total of 29 articles were included in this systematic review. A flow diagram outlining the review process is provided in the Figure.

\section{Study Characteristics}

A total of 19 different activity monitors were evaluated in 29 studies. There were 9 off-the-shelf activity monitors and 10 custom-made devices. The specifications of each device are shown in Appendix 2. Of the 29 articles selected, 6 studies evaluated commercially available monitors with default algorithms for quantifying PA in MWUs [24-29] (Appendix 3), 15 evaluated commercially available monitors with custom algorithms [28-42] (Appendix 4), and 10 evaluated custom-made devices and algorithms [43-52]
(Appendix 5). These activity monitors could be categorized into three types: accelerometer-based, multisensor-based, and others (gyroscope- or HR-based). Among the 29 articles, 14 investigated accelerometer-based monitors ( 1 uniaxial [38] and 13 triaxial [26,32-33,39-42,46-51]), 9 evaluated multisensor-based devices [24-25,2829,31,34-37], 1 evaluated a gyroscope-based monitor [44], and 5 evaluated combinations of any 2 of the 3 types $[27,30,43,45]$. Among all articles, 19 included only MWUs with spinal cord injuries (SCIs) [24,26-29,31,3337,40-41,43-44,46,50-52]; 7 included MWUs with a mix of diagnoses, including SCI, amputation, congenital bone disorder, complex regional pain syndrome, CharcotMarie-Tooth disease, demyelinating disease, dystonia, fibromyalgia, multiple sclerosis, osteoarthritis, osteogenesis imperfecta, poliomyelitis, rheumatoid arthritis, spina 
Table 2.

Findings of fifteen studies that quantified physical activity (PA) in manual wheelchair users with custom models based on commercial monitors.

\begin{tabular}{|c|c|c|c|c|}
\hline Study, Year & Monitors & Criterion Measures & Outcome Measures & Validation Method \\
\hline $\begin{array}{l}\text { García-Massó et al., } \\
2015 \text { [40] }\end{array}$ & ActiGraph GT3X & Cosmed K4b2 ${ }^{*}$ & $\begin{array}{l}\text { PA types } \\
\text { Model } 1 \text { (nondominant wrist): LDA: 85.9\%, QDA: 84.5\%, SVM: 83.2\% } \\
\text { Model } 2 \text { (dominant wrist): LDA: 83.9\%, QDA: 86.7\%, SVM: } 87 \% \\
\text { Model } 3 \text { (both wrists): LDA: 87.1\%, QDA: 90.4\%, SVM: } 86.8 \% \\
\text { Model } 4 \text { (all): LDA: 89.4\%, QDA: 90.7\%, SVM: 93.6\% }\end{array}$ & $\begin{array}{l}80 \% \text { data was used to } \\
\text { train and cross- } \\
\text { validate classifiers; } \\
\text { remaining } 20 \% \text { data } \\
\text { was used to test them }\end{array}$ \\
\hline Learmonth et al., & ActiGraph GT3X & Cosmed K4b2* & $\underline{\text { Energy cost }\left(\mathrm{VO}_{2}\right)}$ & No \\
\hline
\end{tabular}

2016 [39]

Nightingale et al., 2015 [42]

Kooijmans et al., ActiGraph GT3X+ Video analysis 2014 [41]

$\begin{array}{ll}\text { Conger et al., 2014 } & \text { PowerTap SL + } \\ \text { [30] } & \text { Track hub, HR } \\ & \text { strap }\end{array}$

Coutinho et al., 2014 Polar HR monitor [31]

Nightingale et al., ActiGraph GT3X+ Cosmed K4b2* 2014 [32]

García-Massó et al., ActiGraph GT3X Cosmed K4b2* 2013 [33]

Hiremath et al., SW

2013 [34]

vice 2: GENE-

system $^{\dagger}$

Cosmed K4b2*

Cosmed K4b2;
Model 2 (RW): $R=0.95 \pm 0.37, R^{2}=0.90 \pm 0.14$

Model 3 (both wrists): $R=0.94 \pm 0.38, R^{2}=0.88 \pm 0.15$

Energy cost (PAEE)

Model 1 (GT3X at upper arm): MAE: $35.3 \% \pm 30.8 \%, R^{2}=0.46$, SEE $=$ $1.16 \mathrm{kcal} / \mathrm{min}$

Model 2 (GT3X at wrist): MAE: $33.0 \% \pm 39.5 \%, R^{2}=0.67, \mathrm{SEE}=0.91$

$\mathrm{kcal} / \mathrm{min}$

Model 3 (GENEActiv at upper arm): MAE: $20.4 \% \pm 14.3 \%, R^{2}=0.76$,

$\mathrm{SEE}=0.77 \mathrm{kcal} / \mathrm{min}$

Model 4 (GENEActiv at wrist): MAE: $21.0 \% \pm 15.1 \%, R^{2}=0.77$, SEE $=$ $0.75 \mathrm{kcal} / \mathrm{min}$

\section{PA types}

Agreement: $85.2 \%$, range: $76.7 \%$ to $92.3 \%$

Sensitivity: $88.3 \%$, range: $83.1 \%$ to $93.0 \%$

Specificity: $83.3 \%$, range: $72.6 \%$ to $91.2 \%$

Oxycon Mobile

\section{Energy cost (EE)}

Model 1 (using power as a predictor):

$R^{2}=0.48$, SEE: $0.97 \mathrm{kcal} / \mathrm{kg} / \mathrm{h}$, RMSE: $0.97 \mathrm{kcal} / \mathrm{kg} / \mathrm{h}$

Model 2 (using power and speed as predictors): $R^{2}=0.70$, SEE:

$0.74 \mathrm{kcal} / \mathrm{kg} / \mathrm{h}$, RMSE: $0.82 \mathrm{kcal} / \mathrm{kg} / \mathrm{h}$

Model 3 (using power, speed, and HR as predictors): $R^{2}=0.8$, SEE:

$0.48 \mathrm{kcal} / \mathrm{kg} / \mathrm{h}, \mathrm{RMSE}: 0.74 \mathrm{kcal} / \mathrm{kg} / \mathrm{h}$

\section{Energy cost (EE)}

THBI (Using total HR and [measured] distance traveled as predictors): $R^{2}=0.5437$ ( $\left.p<0.001\right), r=0.58$ (95\% CI: 0.36 to 0.74$)$

PCCI (using exercise HR and [measured] propulsion speed as predictors): $R^{2}=0.5295$ ( $\left.p<0.001\right), r=0.59$ (95\% CI: 0.34 to 0.73 )

PCI (using exercise and basal HR and [measured] propulsion speed as predictors): $R^{2}=0.423$ ( $\left.p<0.001\right), r=0.38$ (95\% CI: 0.11 to 0.60$)$

Energy cost (PAEE)

Model 1 (waist): $r=0.73, R^{2}=0.53(p<0.01), \mathrm{SEE}=6.07 \mathrm{~kJ} / \mathrm{min}$

Model 2 (upper arm): $r=0.87, R^{2}=0.75(p<0.01)$, SEE $=4.38 \mathrm{~kJ} / \mathrm{min}$

Model 3 (wrist): $r=0.93, R^{2}=0.86(p<0.01)$, SEE $=3.34 \mathrm{~kJ} / \mathrm{min}$

Energy cost $\left(\mathrm{VO}_{2}\right)$

Model 1 (dominant wrist): $r=0.86$, MSE: 5.16\%, MAE: $1.67 \%$, RMSE: $2.27 \%$

Model 2 (nondominant wrist): $r=0.86$, MSE: 4.98\%, MAE: $1.65 \%$, RMSE: $2.23 \%$

Model 3 (nondominant waist): $r=0.67$, MSE: $10.65 \%$, MAE: $2.39 \%$, RMSE: $3.26 \%$

Model 4 (nondominant side of chest): $r=0.68$, MSE: $10.43 \%$, MAE: 2.41\%, RMSE: $3.23 \%$

Energy cost (EE)

observation (annotation)
QDA: MAE: $17.4 \%$, MSE: $5.3 \%+21.5 \%$

NB: MAE: $18.2 \%$, MSE: $4.6 \% \pm 22.8 \%$

User movements

Overall classification accuracy for 4 activities (resting, propulsion, arm ergometry, and deskwork): QDA: 96.3\%, NB: 94.8\%
No

No

Leave-1-subject-out

cross-validation

Leave-1-subject-out cross-validation

No

20-fold by subject cross-validation

Leave-1-subject-out 
JRRD, Volume 53, Number 6, 2016

Table 2. (cont)

Findings of fifteen studies that quantified physical activity (PA) in manual wheelchair users with custom models based on commercial monitors.

\begin{tabular}{|c|c|c|c|}
\hline Study, Year & Monitors & Criterion Measures & Outcome Measures \\
\hline $\begin{array}{l}\text { Hiremath et al., } \\
2012 \text { [29] }\end{array}$ & SW & Cosmed K4b2 $^{*}$ & $\begin{array}{l}\text { Energy cost (EE) } \\
\text { Model } 1 \text { (general): MSE: 2.3\% (95\% CI: }-1.7 \% \text { to 6.3\%); MAE: } 24.7 \% \\
\text { (95\% CI: } 22.1 \% \text { to } 27.2 \%) \text {; BA: }-1.86,2.60 \mathrm{kcal} / \mathrm{min} \\
\text { Model } 2 \text { (activity-specific overall): MSE: } 4.9 \% \text { (95\% CI: } 2.2 \% \text { to } 7.5 \% \text {; } \\
\text { MAE: } 16.8 \% \text { (95\% CI: } 15.2 \% \text { to } 18.5 \% \text { ); BA: }-1.26,1.96 \mathrm{kcal} / \mathrm{min} \\
\text { General model was developed using all activity data while each of the } \\
4 \text { activity-specific models were developed using resting, deskwork, } \\
\text { wheelchair propulsion, and arm ergometry data }\end{array}$ \\
\hline
\end{tabular}

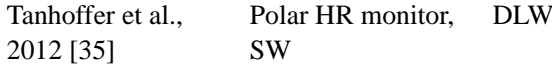

Coulter et al., 2011

[36]

Hiremath et al.,

$2011[28]$

Lee et al., 2010

[37]

Washburn and

Copay, 1999 [38]
RT3

activPAL trio PA monitor

Polar HR monitor

CSA uniaxial accel- $\mathrm{VO}_{2}$ : Aerosport erometer
No. wheel rev: recorded manually by observation. Absolute angle: recorded by handheld digital video recorder and analyzed by Siliconcoach Pro $7^{\S}$ Duration of movement: recorded using timer on video by two independent raters.

Cosmed K4b2*

$\mathrm{VO}_{2}$ during resting: Quark b2 ${ }^{*}$ $\mathrm{VO}_{2}$ during PA: Cosmed K4b2* TEEM 100 Total MAS Polar telemetry transceiver $^{* *}$ Propulsion frequency: counted by investigators

\section{Energy cost (EE)}

Model 1 (SW): TDEE: $R^{2}=0.65, p<0.001$; BA: $-2,156,5,350 \mathrm{~kJ} /$ day or $-0.356,0.888 \mathrm{kcal} / \mathrm{min}$ PAEE: $R^{2}=0.16, p=0.001$; BA: $-5,427,5,338 \mathrm{~kJ} /$ day or -0.901 , $0.886 \mathrm{kcal} / \mathrm{min}$ Model 2 (HR monitoring): TDEE: $R^{2}=0.68, p=0.16$; BA: $-3,598$, $1,878 \mathrm{~kJ} /$ day or $-0.597,0.312 \mathrm{kcal} / \mathrm{min}$ PAEE: $R^{2}=0.30, p=0.07$; BA: $-2,531,3,453 \mathrm{~kJ} /$ day or $-0.420,0.573$ $\mathrm{kcal} / \mathrm{min}$

\section{Wheelchair movements}

Wheel rev: Mean difference: $0.002 \pm 0.016$ rev, Maximum difference: 0.038 rev; MAE: $0.59 \%$; ICC $(2,1)=1.00$ (95\% CI: 1.00 to 1.00 ); BA: $-0.029,0.032 \mathrm{rev}$

Absolute angle of rotation: Mean difference: $0.006 \pm 3.853^{\circ}$, Maximum difference: $8.789^{\circ}$; ICC (2,1) $=0.999$ (95\% CI: 0.999 to 0.999 );

BA: $-7.56,7.55^{\circ}$

\section{User movements}

Duration of movement: Mean difference: $-1.868 \pm 1.392 \mathrm{~s}$, Maximum difference: 7.15 s; ICC $(2,1)=0.981$ (95\% CI: 0.669 to 0.994$)$;

BA: $-4.597,0.861 \mathrm{~s}$

\section{Energy cost (EE)}

Model 1 (RT3 arm): MAE: $12.2 \%$ to $38.1 \%, R^{2}$ : 0.405 to 0.830 , SEE: 0.18 to $0.87 \mathrm{kcal} / \mathrm{min}$

Model 2 (RT3 waist): MAE: $16.1 \%$ to $41.6 \%, R^{2}: 0.247$ to 0.687 , SEE: 0.18 to $1.04 \mathrm{kcal} / \mathrm{min}$

Model 3 (RT3 arm and waist combined): MAE:12.2\% to $38.1 \%, R^{2}$ :

0.405 to 0.864 , SEE: 0.18 to $0.87 \mathrm{kcal} / \mathrm{min}$

Range was among activity trials

\section{Energy cost}

Individual calibration: MAE: $8.38 \% \pm 6.11 \%$, HR ratio and observed MET: $R^{2}=0.90$, observed and predicted MET: $r=0.93$

Group calibration: MAE: $25.79 \% \pm 27.90 \%$, HR ratio and observed MET: $R^{2}=0.59$, observed and predicted MET: $r=0.78$

\section{Energy cost}

Uniaxial CSA count vs EE measured by MAS:

RW: $r=0.52, p<0.01$, SEE: $5.71 \mathrm{~mL} / \mathrm{kg} / \mathrm{min}$;

LW: $r=0.67, p<0.01$, SEE: $4.99 \mathrm{~mL} / \mathrm{kg} / \mathrm{min}$

Uniaxial CSA count vs HR:

RW: $r=0.40, p<0.01$

LW: $r=0.29, p<0.01$

User movements

Propulsion frequency: RW: $r=0.35, p<0.01$; LW: $r=0.26, p<0.01$

\section{Validation Method}

Separate group of

subjects $(n=9)$

No

NA

Separate group of subjects $(n=4)$

Separate set of PA (different than PA performed when developing models) performed by same group of subjects

No 
Table 2. (cont)

Findings of fifteen studies that quantified physical activity (PA) in manual wheelchair users with custom models based on commercial monitors.

Note: An overview of the commercial and custom monitors can be found in Appendix 2 (available online only).

*COSMED; Rome, Italy.

${ }^{\dagger}$ TrueOne; Salt Lake City, Utah.

‡Viasys Healthcare; Hochberg, Germany.

§Siliconcoach; Otago, New Zealand.

"Aerosport, Inc; Ann Arbor, Michigan.

*** Polar Electro, Inc; Finland.

BA = Bland-Altman 95\% limits of agreement, CI = confidence interval, DLW = doubly labeled water, EE = energy expenditure, HR = heart rate, ICC $=$ intraclass correlation coefficient, $\mathrm{LDA}=$ linear discriminant analysis, $\mathrm{LW}=$ left wrist, $\mathrm{MAE}=$ mean absolute error, MAS = metabolic analysis system, MET $=$ metabolic equivalent, MSE = mean signed error, NA = not applicable, NB = Naive Bayes, No. = number, PAEE $=$ Physical Activity Energy Expenditure, PCCI $=$ Propulsion Cardiac Cost Index, PCI = Physiological Cost Index, QDA = quadratic discriminant analysis, rev = revolution, RMSE = root mean square error, RW = right wrist, $\mathrm{SEE}=$ standard error of estimate, $\mathrm{SVM}=$ support vector machine, $\mathrm{SW}=$ SenseWear, TDEE $=$ Total Daily Energy Expenditure, $\mathrm{THBI}=$ Total Heart Beat Index, $\mathrm{VO}_{2}=\mathrm{oxyg}_{\mathrm{g}}$ consumption .

bifida, scoliosis, and traumatic brain injury [25,30,32,3839,42,47]; and 3 did not report the diagnoses of their participants [45,48-49]. The majority of the studies were conducted in a structured laboratory environment. Six studies were conducted in semistructured settings (i.e., the National Veterans Wheelchair Games [47,52]), outpatient care facilities [24], and unstructured environments (i.e., home and a tennis or basketball court [24-26,45,52]).

\section{Outcome Measures, Gold Standards, and Reference Comparisons}

The outcome measures could be categorized into three types: energy cost, wheelchair movements, and user movements. The energy expenditure (EE), oxygen consumption, metabolic equivalent, and HR indexes were frequently reported outcome measures for quantifying the energy cost of PA. Of the 29 selected studies, 14 quantified PA in terms of energy cost [24-25,27-35,3738,50]. Six of the studies reported distance traveled, speed (linear and/or angular), absolute angle of rotation, and number of wheel revolutions as measures of wheelchair movements [36,44-46,48-49]. Eleven of the studies reported propulsion frequency, propulsion force, upper-limb activity counts, duration of user movement, and types of activities as the measures of user movements [24-26,34,36,38,43,46-48,51]. These outcome measures were compared with either gold standards or validated reference measurements.

Indirect calorimetry was the most commonly used gold standard for validating the energy cost from PA monitors. Two studies used doubly labeled water [24,35], and 14 studies used metabolic analysis systems such as Cosmed K4b2 or Quark b2 (COSMED; Rome, Italy), TrueOne 2400 computerized metabolic system (TrueOne; Salt Lake City, Utah), Oxycon mobile (Viasys Healthcare; Hochberg, Ger- many), gas analyzer (AR-1 type-4, Arco System; Chiba, Japan), and Metabolic Analysis System (Aerosport Inc; Ann Arbor, Michigan) as the criterion for measuring energy cost [27-34,37-40,42,50]. Other methods, including video analysis, observation, direct measurements, and motion capture systems, were used as reference comparisons for validating wheelchair and user movements [34,36,41, 43-46,49,51-52]. Six studies used video recording as a reference for distances traveled, propulsion frequency, and duration of movement [36,43,45-46,49,51]. Three studies used direct measurement and observation to record distances traveled, propulsion frequency, and PA types as reference comparisons [34,36,45]. Two studies used SMART $^{\text {Wheel }}$ (Three Rivers Holdings, Inc; Mesa, Arizona) and VICON (Vicon Peak; Lake Forest, California) to measure/calculate the propulsion force, propulsion frequency, and distance traveled [43-44]. In addition, two studies used a validated questionnaire and self-reported PA record as reference comparisons for EE [25-26].

\section{Statistical Analysis and Key Findings}

Among the 18 studies that evaluated the energy cost, 7 reported the mean signed error (MSE) and/or the mean absolute error (MAE). Three of the seven studies used commercial monitors with the default algorithms [2729], and six used commercial monitors with custom algorithms [28-29,33-34,37,42]. The MSE summarizes the accumulated error between the estimation and the criterion over a period of time, where overestimations and underestimations at each instance may cancel each other out. The MAE represents the average of the absolute differences between the estimation and the criterion at each instance. Among the 8 studies, the MSE and MAE of the commercial monitors with the default algorithms ranged from -62.5 to -48.1 percent [29] and from 21.3 
JRRD, Volume 53, Number 6, 2016

Table 3.

Findings of ten studies that quantified physical activity (PA) in manual wheelchair users with custom devices and algorithms.

\begin{tabular}{|c|c|c|c|c|}
\hline Study, Year & Monitor & $\begin{array}{l}\text { Criterion } \\
\text { Measures }\end{array}$ & Outcome Measures & $\begin{array}{c}\text { Validation } \\
\text { Method }\end{array}$ \\
\hline $\begin{array}{l}\text { Hiremath et } \\
\text { al., } 2015 \text { [52] }\end{array}$ & PAMS & $\begin{array}{l}\text { Video record- } \\
\text { ing }\end{array}$ & $\begin{array}{l}\text { PA types } \\
\text { Overall accuracy: Model } 1 \text { (PAMS with accelerometer on arm): } 89.3 \% \\
\text { Model } 2 \text { (PAMS with accelerometer on wrist): } 88.5 \% \\
\text { Model } 3 \text { (wheel rotation monitor): } 65.4 \%\end{array}$ & $\begin{array}{l}\text { Separate set of } \\
\text { data ( } 137 \\
10 \text { min trials) }\end{array}$ \\
\hline
\end{tabular}

Model 4 (accelerometer on arm): $70.4 \%$

Model 5 (accelerometer on wrist): 74.6\%

Kiuchi et al., Motion sensor Gas analyzer 2014 [50]

Ojeda and

Ding, 2014

[43]

Hiremath et al., 2013 [44]

Sindall et al., $2013[45]$

Sonenblum et al., 2012 [46] (triaxial accel- (AR-1 typeerometer and $4^{*}$ ) gyroscope sensor)

Triaxial accel- No. propulerometer, sion: video wheel rotation monitor

Gyroscopebased wheel rotation monitor

GPS tracking device with integrated accelerometer, DL

Triaxial MEMS accelerometer

pulsion frequency: system), track length

Time spent:

Time spent recording; hand-cycling
Energy cost

No

Model 1 (triaxial acceleration only):

LW: $R^{2}=0.64(p<0.001)$, SEE: $0.005 \mathrm{kcal} / \mathrm{min} / \mathrm{kg}$;

RW: $R^{2}=0.68(p<0.001)$, SEE: $0.004 \mathrm{kcal} / \mathrm{min} / \mathrm{kg}$;

LA: $R^{2}=0.66(p<0.001)$, SEE: $0.004 \mathrm{kcal} / \mathrm{min} / \mathrm{kg}$;

RA: $R^{2}=0.82(p<0.001)$, SEE: $0.003 \mathrm{kcal} / \mathrm{min} / \mathrm{kg}$

Model 2 (angular velocity only):

LW: $R^{2}=0.60(p<0.001)$, SEE: $0.005 \mathrm{kcal} / \mathrm{min} / \mathrm{kg}$;

RW: $R^{2}=0.50(p=0.001)$, SEE: $0.005 \mathrm{kcal} / \mathrm{min} / \mathrm{kg}$;

LA: $R^{2}=0.64(p<0.001)$, SEE: $0.0051 \mathrm{kcal} / \mathrm{min} / \mathrm{kg}$;

RA: $R^{2}=0.83(p<0.001)$, SEE: $0.003 \mathrm{kcal} / \mathrm{min} / \mathrm{kg}$

Model 3 (triaxial acceleration and angular velocity):

LW: $R^{2}=0.86(p<0.001)$, SEE: $0.003 \mathrm{kcal} / \mathrm{min} / \mathrm{kg}$; BA: $-0.0083,0.0031$ $\mathrm{kcal} / \mathrm{min} / \mathrm{kg}$;

RW: $R^{2}=0.68(p<0.001)$, SEE: $0.004 \mathrm{kcal} / \mathrm{min} / \mathrm{kg} ;$ BA: $-0.0118,0.0046$

$\mathrm{kcal} / \mathrm{min} / \mathrm{kg}$;

LA: $R^{2}=0.75(p<0.001)$, SEE: $0.004 \mathrm{kcal} / \mathrm{min} / \mathrm{kg}$; BA: $-0.0063,0.0085$

$\mathrm{kcal} / \mathrm{min} / \mathrm{kg}$

RA: $R^{2}=0.87(p<0.001)$, SEE: $0.003 \mathrm{kcal} / \mathrm{min} / \mathrm{kg}$; BA: $-0.0025,0.0081$

$\mathrm{kcal} / \mathrm{min} / \mathrm{kg}$

User movements

NA

No. propulsion: (Arm) MAE: 8.0\% $\pm 7.1 \%$; ICC(3,1): 0.994 (95\% CI: 0.988 to recording; pro- 0.997)

SMART Wheel

(Wrist) MAE: $10.8 \% \pm 9.8 \%$, ICC(3,1): 0.990 (95\% CI: 0.980 to 0.995$)$

(Seat) MAE: $13.4 \% \pm 15.6 \%$, ICC(3,1): 0.984 (95\% CI: 0.972 to 0.991$)$

Propulsion frequency: (Arm) MAE: $12.9 \% \pm 15.1 \%$, ICC(3,1): 0.916 (95\% CI:

0.843 to 0.953 )

(Wrist) MAE: $17.2 \% \pm 19.3 \%$, ICC(3,1): 0.889 (95\% CI: 0.802 to 0.936$)$;

(Seat) MAE: $24.2 \% \pm 16.6 \%$, ICC(3,1): 0.690 (95\% CI: 0.071 to 0.868 )

Distance trav-

Wheelchair movements

eled: VICON

(vision based SMART $^{\text {Wheel }}$, measured total video recording; Distance: tape measure

Distance traveled (range): MAE: $0.17 \%$ to $1.38 \%$, MSE: $-0.94 \%$ to $1.38 \%$, SEM: 0.05 to $0.38 \mathrm{~m}$, ICC(3,1): 0.999 to 1.000

Angular velocity: MAE: $0.03 \%$ to $0.64 \%$, MSE: $0.00 \%$ to $0.06 \%$, SEM: 0.02 to $0.29 \mathrm{rpm}$

Linear speed (range): MAE: $0.66 \%$ to $2.19 \%$, MSE: $-2.19 \%$ to $0.27 \%$, SEM: 0.19 to $0.52 \mathrm{~m} / \mathrm{s}$

\section{Wheelchair movements}

Distance traveled: (GPS) MSE: $5.84 \% \pm 5.53 \%$

(DL-right) MSE: $3.04 \% \pm 7.58 \%$

(DL-left) MSE: $4.15 \% \pm 7.77 \%$

Wheelchair movements

No moving: video Distance measured accuracy: $96 \% \pm 2 \%$ Distance: tape measure (predetermined User movements

Accuracy of identifying moving or being stationary: Accuracy (each time point): moving: $90 \% \pm 6 \%$; stationary: $95 \% \pm 3 \%$

Accuracy (total time): moving: $94 \% \pm 5 \%$; stationary: $93 \% \pm 3 \%$ 
Table 3. (cont)

Findings of ten studies that quantified physical activity (PA) in manual wheelchair users with custom devices and algorithms.

\begin{tabular}{|c|c|c|c|c|}
\hline Study, Year & Monitor & $\begin{array}{l}\text { Criterion } \\
\text { Measures }\end{array}$ & Outcome Measures & $\begin{array}{l}\text { Validation } \\
\text { Method }\end{array}$ \\
\hline $\begin{array}{l}\text { Ding et al., } \\
2011 \text { [47] }\end{array}$ & $\begin{array}{l}\text { Wheelchair } \\
\text { propulsion } \\
\text { monitoring } \\
\text { device: } \\
\text { eWatch }\end{array}$ & $\begin{array}{l}\text { Investigator } \\
\text { hand annota- } \\
\text { tion with stop } \\
\text { watch }\end{array}$ & $\begin{array}{l}\text { User movements } \\
\text { Accuracy of } 3 \text { classification categories: } \\
\text { Self-propulsion: } 84.1 \% \text { to } 88.1 \% \\
\text { External pushing: } 64.2 \% \text { to } 74.6 \% \\
\text { Sedentary activity: } 93.9 \% \text { to } 96 \%\end{array}$ & $\begin{array}{l}\text { Leave-1- } \\
\text { subject-out } \\
\text { cross- } \\
\text { validation }\end{array}$ \\
\hline $\begin{array}{l}\text { Turner, } 2011 \\
\text { [48] }\end{array}$ & PushTracker & $\begin{array}{l}\text { OptiPush Bio- } \\
\text { feedback Sys- } \\
\text { tem }^{\ddagger}\end{array}$ & $\begin{array}{l}\text { Wheelchair movements } \\
\text { Distance traveled: MSE: }-0.1 \% \pm 1 \%, p=0.73 \\
\text { User movements } \\
\text { No. propulsion: MSE: }-1 \% \pm 3 \%, p=0.32 \\
\text { Propulsion frequency: MSE: }-1.7 \% \pm 3.7 \%, p=0.19 \\
\text { Speed: MSE: }-0.8 \% \pm 2.1 \%, p=0.27\end{array}$ & NA \\
\hline $\begin{array}{l}\text { Postma et al., } \\
2005 \text { [51] }\end{array}$ & $\begin{array}{l}\text { ADXL202 } \\
\text { piezo-resistive } \\
\text { accelerometer }\end{array}$ & $\begin{array}{l}\text { Video record- } \\
\text { ing using } \\
\text { handheld } \\
\text { camera }\end{array}$ & $\begin{array}{l}\text { User movements } \\
\text { Classification of wheelchair propulsion and nonwheelchair } \\
\text { propulsion activities. } \\
\text { Agreement: } 92 \% \text { (range: } 87 \% \text { to } 96 \% \text { ) } \\
\text { Sensitivity: } 87 \% \text { (range: } 76 \% \text { to } 99 \% \text { ) } \\
\text { Specificity: } 92 \% \text { (range: } 85 \% \text { to } 98 \% \text { ) } \\
\text { Duration of wheelchair propulsion: Estimation error (range): } \\
2 \% \text { to } 29 \% \text { (i.e., } 22 \text { to } 283 \text { s) } \\
\text { Range was among subjects }\end{array}$ & No \\
\hline $\begin{array}{l}\text { Moss et al., } \\
2003 \text { [49] }\end{array}$ & $\begin{array}{l}\text { Telemetry- } \\
\text { based } \\
\text { velcometer }\end{array}$ & $\begin{array}{l}\text { Video record- } \\
\text { ing, kinematic } \\
\text { analysis }\end{array}$ & $\begin{array}{l}\text { Wheelchair movements } \\
\text { Average RMS deviation: } 0.06 \pm 0.01 \mathrm{~m} / \mathrm{s} \text { (propel at } 1 \mathrm{~m} / \mathrm{s}), 0.27 \pm 0.05 \mathrm{~m} / \mathrm{s} \\
\text { (propel at } 5 \mathrm{~m} / \mathrm{s}), 0.48 \pm 0.16 \mathrm{~m} / \mathrm{s} \text { (propel at } 9 \mathrm{~m} / \mathrm{s}), 0.27 \pm 0.07 \mathrm{~m} / \mathrm{s} \text { (accelera- } \\
\text { tion), } 0.35 \pm 0.06 \mathrm{~m} / \mathrm{s} \text { (deceleration) } \\
\text { Speed: Disc wheel: MSE: } 0.0 \% \pm 0.17 \%(95 \% \mathrm{CI}:-0.34 \% \text { to } 0.34 \%) \\
\text { Spoke wheel: MSE: } 0.0 \% \pm 0.41 \%(95 \% \mathrm{CI}:-0.82 \% \text { to } 0.82 \%)\end{array}$ & NA \\
\hline \multicolumn{5}{|c|}{$\begin{array}{l}\text { Note: An overview of the commercial and custom monitors can be found in Appendix } 2 \text { (available online only). } \\
{ }^{*} \text { Arco System; Chiba, Japan. } \\
{ }^{\dagger} \text { Three Rivers Holdings, LLC; Mesa, Arizona. } \\
\text { †S Sun Components; Milwaukee, Wisconsin. } \\
\text { BA = Bland-Altman 95\% limits of agreement, CI = confidence interval, DL = Datalogger, ICC = intraclass correlation coefficient, LA = left arm, LW = left wrist } \\
\text { MAE = mean absolute error, MSE = mean signed error, NA = not applicable, No. = number, PAMS = Physical Activity Monitoring System, RA = right arm, RMS } \\
\text { root mean square, RW = right wrist, SEE = standard error of estimate, SEM = standard error of the mean. }\end{array}$} \\
\hline
\end{tabular}

[28] to 125.8 percent [27], respectively. On the other hand, the MSE and MAE of the commercial monitors with the custom algorithms ranged from -41.0 to 50.2 percent [34] and from 1.65 [33] to 81.6 percent [37], respectively (Tables 1 and 2). For MSE, the negative number indicates overestimation while the positive number indicates underestimation. In addition, 13 out of 18 studies reported the correlation coefficient (Pearson or Spearman) between the estimation and the criterion [2425,27-28,30-33,35,37-39,42]. The correlation coefficient between the estimated energy cost by the commercial monitors with the default algorithms and the criterion ranged from 0.35 [25] to 0.84 [27] (Table 1), while the correlation coefficient between the estimated energy cost by the commercial monitors with the custom algorithms and the criterion ranged from 0.40 [35] to 0.95 [39] (Table 2).

The Bland-Altman plot and the intraclass correlation coefficient (ICC) were also often reported for evaluating the energy cost. The Bland-Altman plot assesses the agreement between the estimated values and the criterion measures [53]. It illustrates the systematic difference between the estimated values and criterion measures as well as outliers [53]. The mean difference represents the estimated bias, while the 95 percent limits of agreement provide an idea of how far apart two measures are likely to be for most individuals. Three studies constructed the Bland-Altman plots and reported the mean difference and the 95 percent limits of agreement (Tables 1-3) $[29,35,50]$. The ICC, on the other hand, is a reliability 


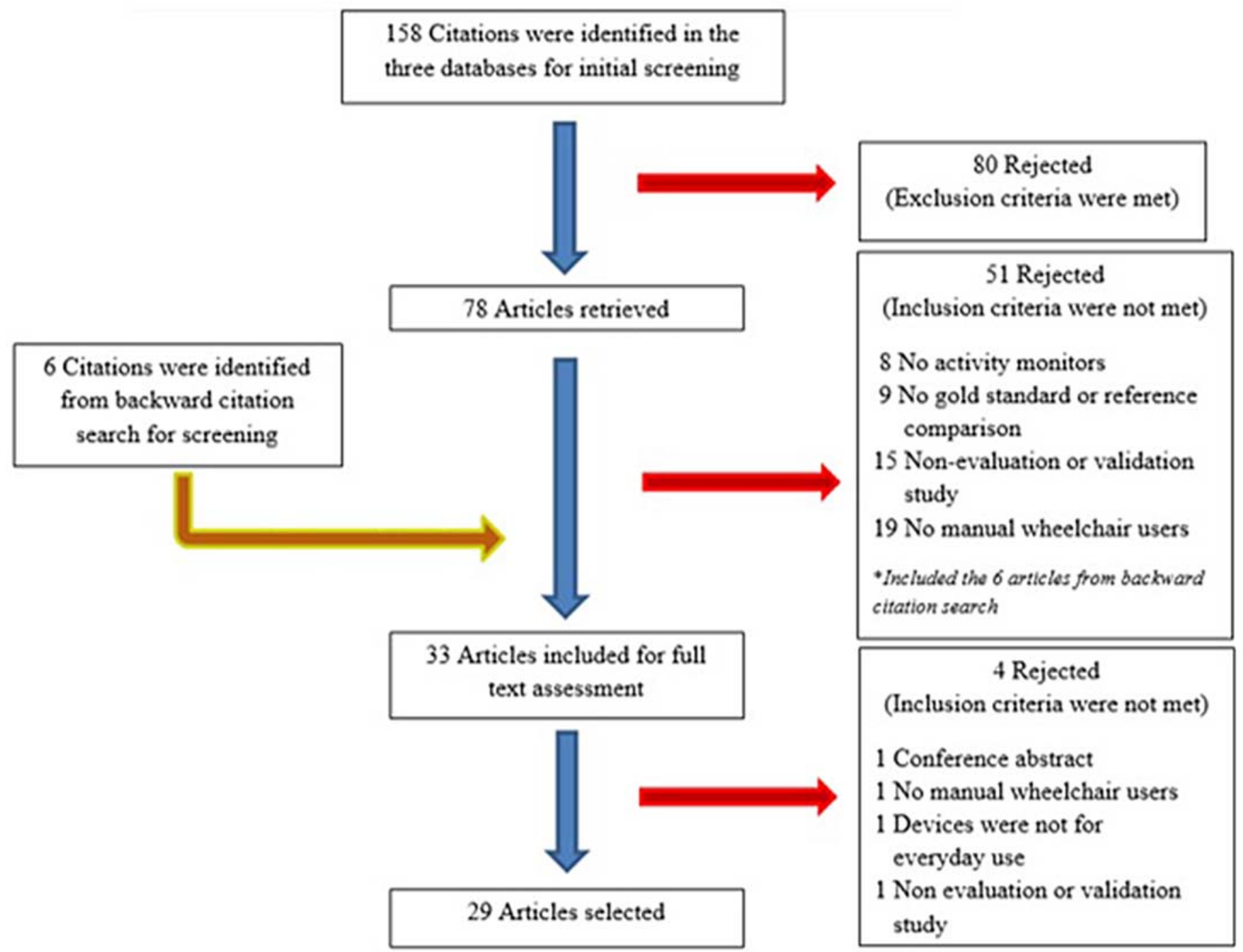

Figure.

Study selection flow diagram.

measure that considers the systematic differences in paired observations [54]. In other words, the ICC represents the extent to which monitors produce the same rank orders in the outcomes as the criterion measures. One study reported the ICC $(3,1)$ between the energy cost estimated by the commercial monitors, i.e., the SenseWear (Jawbone: San Francisco, California) and RT3 (Stayhealthy Inc; Monrovia, California), with the default algorithms, and the criterion measures were 0.62 and 0.64 , respectively, showing a medium strength of agreement [27] (Table 1).

For quantifying wheelchair movements, various statistical analyses such as the MSE, the correlation coeffi- cient, the Bland-Altman plot, and the ICC were used. A total of 6 studies measured wheelchair movements in MWUs in terms of the distance traveled, linear and/or angular speed, absolute angle of rotation, and number of wheel revolutions [34,36,45-46,48-49]. Coulter et al. reported that the MAE, $\operatorname{ICC}(2,1)$ and the Bland-Altman 95 percent limits of agreement for the number of wheel revolutions were 0.59 percent, 1.00 , and -0.029 to 0.032 revolutions, respectively [36]. In addition, Coulter et al. found the $\operatorname{ICC}(2,1)$ and Bland-Altman 95 percent limits of agreement for the absolute angle of rotation were 0.999 and $-7.56^{\circ}$ to $7.55^{\circ}$, respectively [36]. Five studies [44-46,48-49] evaluated the custom devices/algorithms, 
and the MSE and MAE for distance traveled ranged from -11.4 to 19.7 percent [45] and from 0.17 to 1.38 percent [44], respectively (Table 3). Additionally, the MSE and MAE for linear and angular speed ranged from -2.19 [44] to 0.82 percent [49] and from 0.03 to 2.19 percent [44], respectively (Table 3).

A total of 14 studies quantified user movements in terms of duration, propulsion frequency, number of propulsions, and PA types performed [24-26,34,36,38,4041,43,46-48,51-52]. Two studies evaluated the commercial monitors with default algorithms on the duration of user movement using validated questionnaires (Table 1). The correlation coefficients between the monitors' outputs and the reference measurements were 0.60 [26] and 0.53 [25], respectively. Two studies evaluated the commercial monitors with the custom algorithms. One study reported that the Bland-Altman 95 percent limits of agreement and an $\operatorname{ICC}(2,1)$ were -4.597 to $0.861 \mathrm{~s}$ and 0.981 , respectively [36], for the duration of user movement. Another study found the correlation coefficient between the estimated and the measured propulsion frequency were 0.26 and 0.35 when the device was worn on the left and right wrist, respectively [38] (Table 2). The MAE and ICC $(3,1)$ for the number of propulsions ranged from 8.0 to 13.4 percent and from 0.984 to 0.994 , respectively [43], while that for the propulsion frequency ranged from 12.9 to 24.2 percent and from 0.690 to 0.916, respectively [43] (Table 3). Three studies evaluated the commercial monitors with the custom algorithms in classifying PA performed by MWUs [40-41,52]. One reported that the percent accuracy of classifying four types of PA (resting, propulsion, arm ergometry, and deskwork) were 94.8 percent and 93.6 percent while using naïve Bayes and quadratic discriminant analysis algorithms, respectively [34] (Table 2). A different study reported a percent accuracy of 89.4 percent, 90.7 percent, and 93.6 percent for classifying five types of PA (sedentary, transfers, housework, locomotion, and moderate PA) using linear discriminant analysis, quadratic discriminant analysis, and support vector machine, respectively [40] (Table 2). Another study reported a sensitivity of 88.3 percent and a specificity of 83.3 percent for classifying two types of PA (self-propelled wheelchair driving and other activities) [41] (Table 2). Two studies examined custom devices/algorithms and reported an accuracy ranging from 87 [51] to 94 percent [46] for recognizing moving versus being stationary (Table 3). Lastly, two studies examining custom devices/algorithms showed an accuracy of 64.2 to
96.0 percent for detecting three types of PA (selfpropulsion, external pushing, and sedentary activities) [47], and an accuracy of 89.3 percent for identifying seven types of PA (resting, arm ergometry, household activities, wheelchair propulsion, being pushed in chair, wheelchair basketball, and activities that may involve wheelchair movements) in MWUs [52], respectively (Table 3).

\section{DISCUSSION}

\section{Validity of Activity Monitors in Manual Wheelchair Users}

Overall, the commercial monitors with the default algorithms were generally not suitable for tracking PA in MWUs, as the default algorithms failed to detect wheelchair-based activities that were not usually found in the ambulatory population. When examining the performance of commercial monitors in the ambulatory population, we found that their accuracy (MSE) in estimating energy cost was 12.07 percent (95\% CI: $-18.28 \%$ to $5.85 \%$ ) in uniaxial accelerometer-based monitors, 6.85 percent (95\% CI: $-4.49 \%$ to $18.20 \%$ ) in triaxial accelerometer-based monitors, and 3.64 percent (95\% CI: $-1.70 \%$ to $8.97 \%$ ) in multisensor devices [21]. In general, the estimation errors of activity monitors in the ambulatory population fell within 20 percent when compared with criterion measures, and the pooled Pearson correlation was 0.68 (95\% CI: 0.56 to 0.77) [21]. In contrast, when applying the commercial monitors with the default algorithms to people who rely on wheelchairs for mobility, the MSE was -55.3 percent (95\% CI: $-62.5 \%$ to $-48.1 \%$ ) for multisensor devices [29]. The MAE was 21.3 to 55.2 percent and 24.4 to 125.8 percent, respectively, for triaxial accelerometer-based and multisensor devices [27-29]. Additionally, the Pearson correlation ranged from 0.35 [25] to 0.83 [24]. With the correction on the default algorithms in commercial monitors for wheelchair users, we have observed a general reduction in the estimation errors. The MSE was 4.6 percent (95\% CI: $-41.0 \%$ to $50.2 \%$ ) for multisensor devices with the custom algorithms [34]. The MAE was 1.65 [33] to 41.6 percent [28] and 16.8 to 24.7 percent [29] for triaxial accelerometer-based and multisensor devices with the custom models, respectively, and the Pearson correlation ranged from 0.38 [31] to 0.93 [32]. Despite the improved accuracy with the correction on the default algorithms and custom devices/algorithms, the performance of the activity monitors in tracking energy cost of MWUs was not on par 
with the accuracy for the ambulatory population. A wide variability was found in study results because of the lack of consistency in experimental protocols and evaluation measures used in different studies. Therefore, it was difficult to compare individual monitors/algorithms across different studies and conclude which specific monitor was better in quantifying energy cost for MWUs. The accuracy in tracking energy cost by the portable monitors is particularly important for weight-management interventions, in which users can use the estimated energy cost for attaining and maintaining body weight, and for better understanding dose-response relation between PA and health in this population [55-56].

Aside from the energy cost, PA monitors were also used to quantify wheelchair and user movements. For the wheelchair movements, the commercial monitors with custom algorithms showed a MAE $<1$ percent and ICC $(2,1)$ of 0.999 for measuring wheel revolutions and absolute angle of rotation [36]. In addition, the custom devices/algorithms showed a MSE < 5 percent for measuring speed and distance traveled [48-49]. For the user movements, the commercial monitors with default algorithms showed a moderate strength of correlation with the reference comparison on the duration of movements [25]. The custom devices/algorithms, on the other hand, showed an accuracy of 87 percent for tracking the duration of movements [51] and an accuracy as high as 95 percent for propulsion frequency [48]. Although not all commercial monitors were designed to quantify wheelchair and user movements, with some adaptations, commercial monitors with custom algorithms and custom monitors were able to track these variables with fairly good accuracy. These measurements were insightful in providing additional information about wheelchair usage and frequency of upper-limb movements that may help researchers to further investigate the relationship and find the balance between PA and injuries resulting from upper-limb overuse.

\section{Limitations of Evaluation Studies}

Different studies selected different methods and measures to examine the validity of the activity monitors. MSE, MAE, ICC, correlation coefficient, and BlandAltman 95 percent limits of agreement were the commonly used assessment measures among studies; however, they were not reported in the same way or not all of them were reported. MSE and MAE are measurements of prediction errors. Since a small MSE can result from cancelation of under- and overestimations over a period of time, it is necessary to state the length of time in which the MSE was calculated and the types of PA involved during this period of time. The MAE, which represents the absolute difference between two measures, should be reported with MSE to give a more complete idea of the performance of the monitors. In addition, some studies reported the range while the others reported 95 percent CIs with the average MSE, MAE, ICC, correlation coefficient, and Bland-Altman limits of agreement. The various analytical methods and styles of presenting results made it challenging for researchers to compare and contrast across different studies, and it also prevented researchers from pooling results from all studies to make a comprehensive observation. Therefore, a standardized way to report findings should be established for validating monitors. It is recommended that accuracy measures such as MSE and MAE along with reliability measures such as ICC and Bland-Altman 95 percent limits of agreement be included as they provide a comprehensive evaluation on the overall performance of the monitors.

In addition to the analytical limitations, the studies included in this review were generally limited in terms of the generalizability of their results. Most studies tended to evaluate the devices/algorithms with a homogeneous group of participants. Nineteen out of twenty-nine studies included only MWUs with SCI. According to a disability statistics report in 2002, cerebrovascular disease, or stroke, is the leading condition associated with wheelchair use, accounting for 11.1 percent of all wheelchair users [10]. Arthritis, multiple sclerosis, and amputation accounted for 10.4 percent, 5.0 percent, and 3.7 percent respectively [10]. Paraplegia from SCI at or below T1 accounts for 3.6 percent of all wheelchair users [10]. Other diagnoses also resulting in wheelchair use include, but are not limited to, orthopedic impairment of the lower limb (3.6\%), heart disease (3.3\%), cerebral palsy (3.1\%), rheumatoid arthritis (3.0\%), and diabetes (2.4\%) [10]. So far, the validity of the use of monitors in MWUs is limited to those with SCI (Appendixes 3-5). Further investigations on MWUs with other diagnoses will greatly improve the external validity of the studies. In addition, many studies adopted stringent study protocols, which also reduced the external validity of the results. The controlled environment and structured activity trials may not reflect the everyday life of a MWU. Having less structured PA protocols that include a larger variety of activities such as sports/recreational and free-living activities could potentially improve the generalizability of the 
results and may benefit the application of PA monitors for everyday use.

The quality of the evaluations was also limited by the criterion measures and validation methods chosen. Portable metabolic cart and doubly labeled water were two of the most commonly used criterion measures for evaluating the performance of activity monitors in quantifying the energy cost. The portable metabolic cart is a wearable device, but a facial mask is needed to collect gas samples, which limits the types of activities people can perform [57], whereas doubly labeled water requires urine or saliva specimens before and after drinking an initial dose of ${ }^{2} \mathrm{H}_{2}{ }^{18} \mathrm{O}$ [57]. The portable metabolic cart provides breath-by-breath energy cost, while the doubly labeled water provides an overall energy cost over the entire monitoring period. Depending on the purpose of the study, researchers should choose the appropriate criterion measures for evaluation. Furthermore, the validation method was important when evaluating the predictive abilities of the devices and algorithms [58-59]. There are three levels of validation. The highest level is achieved by using a completely different set of samples for testing and evaluation [60]. Then followed by partitioning the sample into subsets (also known as cross-validation); one subset is used for algorithms, devices training, and development while the other is for testing and validation [59]. The lowest level of validation uses the same sample for both training and testing, which does not indicate the predictive performance of the new algorithms on unseen samples as the other two levels of validation do. Without properly conducted validation, it was difficult to conclude whether the validity observed was due to overfitting [59-60].

\section{Future Development of Physical Activity Monitors and Potential Applications}

Almost all of the activity monitors investigated in this review focused on the quantity of movement in terms of the intensity levels and durations of activities. However, the quality of the movement is equally important. For example, muscular imbalance in the shoulder has been identified as a source of pain and injury in MWUs [61]. Therefore, activity monitors that measure the quality of the movements during PA, i.e., the physical forms of movements and the frequency of different forms performed, may provide additional clinical insights. Previous studies found that manual wheelchair usage is highly associated with upper-limb repetitive strain injury (RSI)
[62-64]. When MWUs increase the amount of PA they perform every day for the purpose of lowering the risks of developing chronic illnesses such as cardiovascular diseases, obesity, and diabetes, they may be at risk of developing another chronic condition, i.e., RSI. Studies have shown improper propulsion patterns and transfer techniques and some specific actions that cause impingement may increase the incidences of RSI at the wrist, elbow, and shoulder joints [65-67]. The chronic pain from RSIs can prevent MWUs from further participation in PA, as well as create a barrier that requires them to become more sedentary than before. Characterizing the forms of movements and assessing their appropriateness during PA can be critical for allowing MWUs to safely participate in regular PA and develop a healthier lifestyle without risking upper-limb RSIs.

\section{Limitations of Review}

This systematic review included only journal and conference articles found in three databases: PubMed, IEEE, and Scopus. It is possible that some relevant studies were not included. The commercial activity monitors evaluated in the selected articles were only a subset of those that are currently available on the market. These issues contributed to a mild selection bias. Moreover, due to the heterogeneity of the selected studies, including differences in study protocols, outcome measures, and analytical methods, the results could not be pooled to obtain a better accuracy estimation of the PA monitors for MWUs.

\section{CONCLUSIONS}

The commercial monitors with the default algorithms were not accurate in estimating energy cost in MWUs and thus are not suitable for these individuals to track their everyday energy output. While the commercial monitors with the custom algorithms or the custom devices did better, their performance was not on par with those used in the ambulatory population. Nonetheless, the commercial monitors with custom algorithms or custom monitors showed fair accuracy in measuring wheelchair and user movements. Adaptations to the commercial monitors or development of monitors specifically for MWUs are needed for quantifying PA in this population. The external validity of the selected studies is also limited because of the homogeneous samples and stringent testing and evaluation 
protocols. Evaluations of activity monitors used in real-life situations are scarce. Further validation studies in MWUs with various diagnoses performing free-living activities in more realistic scenarios are needed.

\section{ACKNOWLEDGMENTS}

\author{
Author Contributions: \\ Established systematic search strategy and eligibility criteria for \\ selecting articles: K. Tsang, D. Ding. \\ Reviewed systematic search strategy: B. E. Dicianno. \\ Performed search based on search strategy: K. Tsang. \\ Screened and selected articles based on eligibility criteria: K. Tsang, \\ S. V. Hiremath, D. Ding. \\ Appraised selected articles: K. Tsang, S. V. Hiremath, D. Ding. \\ Drafting of manuscript: K. Tsang. \\ Editing manuscript: S. V. Hiremath, T. M. Crytzer, B. E. Dicianno, \\ D. Ding. \\ Supervised review article: D. Ding. \\ Financial Disclosures: The authors have declared that no competing \\ interests exist. \\ Funding/Support: This material was based on work supported by the \\ Rehabilitation Engineering Research Center on Interactive Exercise \\ and Recreational Technologies and Exercise Physiology Benefitting \\ People with Disabilities (RecTech) Project R1 (grant \#H133E120005) \\ from the Department of Education, National Institute on Disability \\ and Rehabilitation Research.
}

\section{REFERENCES}

1. World Health Organization (WHO). Global strategy on diet, physical activity and health [Internet]. Geneva (Switzerland): WHO; [cited 2016 Mar 1]. Available from: http://www.who.int/dietphysicalactivity/pa/en/

2. Centers for Disease Control and Prevention (CDC). Rising health care costs are unsustainable [Internet]. Atlanta (GA): CDC; [updated 2013 Oct 23; cited 2016 Mar 7]. Available from: http://www.cdc.gov/chronicdisease/

3. Dixon-Ibarra A, Horner-Johnson W. Disability status as an antecedent to chronic conditions: National Health Interview Survey, 2006-2012. Prev Chronic Dis. 2014;11(130251): 130251. [PMID:24480632] http://dx.doi.org/10.5888/pcd11.130251

4. HealthyPeople.gov. Physical activity [Internet]. Washington (DC): U.S. Department of Health and Human Services; [cited 2016 Mar 7]. Available from:

http://www.healthypeople.gov/2020/topicsobjectives2020/ overview.aspx?topicid $=33$

5. Centers for Disease Control and Prevention (CDC), Division of Nutrition, Physical Activity, and Obesity, National Center for Chronic Disease Prevention and Health Promo- tion. Physical activity and health-The benefits of physical activity [Internet]. Atlanta (GA): CDC; [updated 2015 Jun 4; cited 2016 Mar 7]. Available from: http://www.cdc.gov/ physicalactivity/everyone/health/

6. National Institutes of Health (NIH). Benefits of physical activity [Internet]. Bethesda (MD): NIH; 2011 [updated 2015 Oct 29; cited 2016 Mar 7]. Available from: https://www.nhlbi.nih.gov/health/health-topics/topics/ phys/benefits

7. Go A. Physical activity for people with mobility issues or disabilities [Internet]. 2014 [cited 2016 Mar 1]. Available from: http://www.healthyalberta.com/626.htm

8. National Center on Health, Physical Activity and Disability (NCHPAD). Exercise guidelines for people with disabilities [Internet]. Birmingham (AL): NCHPAD; 2014 [cited 2016 Mar 1]. Available from: http://www.nchpad.org/14/ 74/Exercise $\sim$ Guidelines $\sim$ for $\sim$ People $\sim$ with $\sim$ Disabilities

9. van der Woude LH, Dallmeijer AJ, Janssen TW, Veeger D. Alternative modes of manual wheelchair ambulation: An overview. Am J Phys Med Rehabil. 2001;80(10):765-77. [PMID:11562560] http://dx.doi.org/10.1097/00002060-200110000-00012

10. Kaye H, Kang T, LaPlante MP. Wheelchair use in the United States. San Francisco (CA): The Disability Statistics Center: School of Nursing, University of California; 2002.

11. Greer N, Brasure M, Wilt TJ. Wheeled mobility (wheelchair) service delivery: Comparative effectiveness technical briefs, No. 9 [Internet]. Rockville (MD): Agency for Healthcare Research and Quality; 2012 [cited 2015 Jul 15]. Available from:

http://www.ncbi.nlm.nih.gov/books/NBK83954/

12. Warms CA, Whitney JD, Belza B. Measurement and description of physical activity in adult manual wheelchair users. Disabil Health J. 2008;1(4):236-44.

[PMID:21122734] http://dx.doi.org/10.1016/j.dhjo.2008.07.002

13. Centers for Disease Control and Prevention (CDC). Adults with disabilities-Vital signs for disease control and prevention factsheet [Internet]. Atlanta (GA): CDC; 2014 [updated 2014 May 6; cited 2014 Jun 18]. Available from: http://www.cdc.gov/vitalsigns/disabilities/

14. Durstine J, Gordon B, Wang Z, Luo X. Chronic disease and the link to physical activity. J Sport Health Sci. 2013; 2(1):3-11. http://dx.doi.org/10.1016/j.jshs.2012.07.009

15. American Physical Therapy Association. Guide to physical therapist practice. Second edition. Phys Ther. 2001; 81(1):9-746.

16. LaPorte RE, Montoye HJ, Caspersen CJ. Assessment of physical activity in epidemiologic research: Problems and prospects. Public Health Rep. 1985;100(2):131-46.

[PMID:3920712] 
17. Prince SA, Adamo KB, Hamel ME, Hardt J, Connor Gorber S, Tremblay M. A comparison of direct versus selfreport measures for assessing physical activity in adults: A systematic review. Int J Behav Nutr Phys Act. 2008; 5(1):56. [PMID:18990237] http://dx.doi.org/10.1186/1479-5868-5-56

18. Adams SA, Matthews CE, Ebbeling CB, Moore CG, Cunningham JE, Fulton J, Hebert JR. The effect of social desirability and social approval on self-reports of physical activity. Am J Epidemiol. 2005;161(4):389-98. [PMID:15692083] http://dx.doi.org/10.1093/aje/kwi054

19. Brown WJ, Trost SG, Bauman A, Mummery K, Owen N. Test-retest reliability of four physical activity measures used in population surveys. J Sci Med Sport. 2004;7(2): 205-15. [PMID:15362316] http://dx.doi.org/10.1016/S1440-2440(04)80010-0

20. Shephard RJ. Limits to the measurement of habitual physical activity by questionnaires. Br J Sports Med. 2003; 37(3):197-206, discussion 206. [PMID:12782543] http://dx.doi.org/10.1136/bjsm.37.3.197

21. Van Remoortel H, Giavedoni S, Raste Y, Burtin C, Louvaris Z, Gimeno-Santos E, Langer D, Glendenning A, Hopkinson NS, Vogiatzis I, Peterson BT, Wilson F, Mann B, Rabinovich R, Puhan MA, Troosters T; PROactive consortium. Validity of activity monitors in health and chronic disease: A systematic review. Int J Behav Nutr Phys Act. 2012;9(1):84. [PMID:22776399]

http://dx.doi.org/10.1186/1479-5868-9-84

22. Brown W, Bauman A, Chey T, Trost S, Mummery K. Comparison of surveys used to measure physical activity. Aust N Z J Public Health. 2004;28(2):128-34.

[PMID:15233351]

http://dx.doi.org/10.1111/j.1467-842X.2004.tb00925.x

23. Berlin JE, Storti KL, Brach JS. Using activity monitors to measure physical activity in free-living conditions. Phys Ther. 2006;86(8):1137-45. [PMID:16879047]

24. Tanhoffer RA, Tanhoffer AI, Raymond J, Johnson NA, Hills AP, Davis GM. Energy expenditure in individuals with spinal cord injury quantified by doubly labeled water and a multi-sensor armband. J Phys Act Health. 2015; 12(2):163-70. [PMID:24770697]

http://dx.doi.org/10.1123/jpah.2013-0190

25. Perez-Tejero J, Garcia-Hernandez JJ, Coteron J, BenitoPeinado PJ, Sampedro-Molinuevo J. Medicion de los niveles de actividad fisica en personas con discapacidad fisica mediante acelerometria y cuestionario. Archivos de Medicina del Deporte. 2012;25(147):517-26. Spanish.

26. Warms CA, Belza BL. Actigraphy as a measure of physical activity for wheelchair users with spinal cord injury. Nurs Res. 2004;53(2):136-43. [PMID:15084999] http://dx.doi.org/10.1097/00006199-200403000-00010
27. Hiremath SV, Ding D. Evaluation of activity monitors in manual wheelchair users with paraplegia. J Spinal Cord Med. 2011;34(1):110-17. [PMID:21528634] http://dx.doi.org/10.1179/107902610X12911165975142

28. Hiremath S, Ding D. Regression equations for RT3 activity monitors to estimate energy expenditure in manual wheelchair users. Conf Proc IEEE Med Biol Soc. 2011;2011: 7348-51.

29. Hiremath SV, Ding D, Farringdon J, Cooper RA. Predicting energy expenditure of manual wheelchair users with spinal cord injury using a multisensor-based activity monitor. Arch Phys Med Rehabil. 2012;93(11):1937-43.

[PMID:22609119]

http://dx.doi.org/10.1016/j.apmr.2012.05.004

30. Conger SA, Scott SN, Bassett DR Jr. Predicting energy expenditure through hand rim propulsion power output in individuals who use wheelchairs. Br J Sports Med. 2014; 48(13):1048-53. [PMID:24825852]

http://dx.doi.org/10.1136/bjsports-2014-093540

31. Coutinho AC, Neto FR, Beraldo PS. Validity of heart rate indexes to assess wheeling efficiency in patients with spinal cord injuries. Spinal Cord. 2014;52(9):677-82.

[PMID:25000953]

http://dx.doi.org/10.1038/sc.2014.107

32. Nightingale TE, Walhim JP, Thompson D, Bilzon JL. Predicting physical activity energy expenditure in manual wheelchair users. Med Sci Sports Exerc. 2014;46(9):1849-58. [PMID:25134004] http://dx.doi.org/10.1249/MSS.0000000000000291

33. García-Massó X, Serra-Añó P, García-Raffi LM, SánchezPérez EA, López-Pascual J, Gonzalez LM. Validation of the use of Actigraph GT3X accelerometers to estimate energy expenditure in full time manual wheelchair users with spinal cord injury. Spinal Cord. 2013;51(12):898-903. [PMID:23999111] http://dx.doi.org/10.1038/sc.2013.85

34. Hiremath SV, Ding D, Farringdon J, Vyas N, Cooper RA. Physical activity classification utilizing SenseWear activity monitor in manual wheelchair users with spinal cord injury. Spinal Cord. 2013;51(9):705-9. [PMID:23689386] http://dx.doi.org/10.1038/sc.2013.39

35. Tanhoffer RA, Tanhoffer AI, Raymond J, Hills AP, Davis GM. Comparison of methods to assess energy expenditure and physical activity in people with spinal cord injury. J Spinal Cord Med. 2012;35(1):35-45. [PMID:22330189] http://dx.doi.org/10.1179/2045772311Y.0000000046

36. Coulter EH, Dall PM, Rochester L, Hasler JP, Granat MH. Development and validation of a physical activity monitor for use on a wheelchair. Spinal Cord. 2011;49(3):445-50. [PMID:20856261] http://dx.doi.org/10.1038/sc.2010.126 
37. Lee M, Zhu W, Hedrick B, Fernhall B. Estimating MET values using the ratio of HR for persons with paraplegia. Med Sci Sports Exerc. 2010;42(5):985-90.

[PMID:19997011]

http://dx.doi.org/10.1249/MSS.0b013e3181c0652b

38. Washburn R, Copay AG. Assessing physical activity during wheelchair pushing: Validity of a portable accelerometer. Adapt Phys Activ Q. 1999;16(3):290-99.

http://dx.doi.org/10.1123/apaq.16.3.290

39. Learmonth YC, Kinnett-Hopkins D, Rice IM, Dysterheft JL, Motl RW. Accelerometer output and its association with energy expenditure during manual wheelchair propulsion. Spinal Cord. 2016;54(2):110-14. [PMID:25777327] http://dx.doi.org/10.1038/sc.2015.33

40. García-Massó X, Serra-Añó P, Gonzalez LM, Ye-Lin Y, Prats-Boluda G, Garcia-Casado J. Identifying physical activity type in manual wheelchair users with spinal cord injury by means of accelerometers. Spinal Cord. 2015; 53(10):772-77. [PMID:25987002]

http://dx.doi.org/10.1038/sc.2015.81

41. Kooijmans H, Horemans HL, Stam HJ, Bussmann JB. Valid detection of self-propelled wheelchair driving with two accelerometers. Physiol Meas. 2014;35(11):2297-2306.

[PMID:25340938]

http://dx.doi.org/10.1088/0967-3334/35/11/2297

42. Nightingale TE, Walhin JP, Thompson D, Bilzon JL. Influence of accelerometer type and placement on physical activity energy expenditure prediction in manual wheelchair users. PLoS ONE. 2015;10(5):e0126086.

[PMID:25955304]

http://dx.doi.org/10.1371/journal.pone.0126086

43. Ojeda M, Ding D. Temporal parameters estimation for wheelchair propulsion using wearable sensors. Biomed Res Int. 2014(2014):645284.

44. Hiremath SV, Ding D, Cooper RA. Development and evaluation of a gyroscope-based wheel rotation monitor for manual wheelchair users. J Spinal Cord Med. 2013;36(4): 347-56. [PMID:23820150] http://dx.doi.org/10.1179/2045772313Y.0000000113

45. Sindall P, Lenton JP, Whytock K, Tolfrey K, Oyster ML, Cooper RA, Goosey-Tolfrey VL. Criterion validity and accuracy of global positioning satellite and data logging devices for wheelchair tennis court movement. J Spinal Cord Med. 2013;36(4):383-93. [PMID:23820154] http://dx.doi.org/10.1179/2045772312Y.0000000068

46. Sonenblum SE, Sprigle S, Caspall J, Lopez R. Validation of an accelerometer-based method to measure the use of manual wheelchairs. Med Eng Phys. 2012;34(6):781-86. [PMID:22698978] http://dx.doi.org/10.1016/j.medengphy.2012.05.009

47. Ding D, Hiremath SV, Chung Y, Cooper RA. Detection of wheelchair user activities using wearable sensors. In:
Stephanidis C, editor. Universal Access in HumanComputer Interaction. Context Diversity: Part III (Lecture Notes in Computer Science). 6th International Conference on Universal Access in Human-Computer Interaction, UAHCI 2011; 2011 Jul 9-14; Orlando, FL. New York (NY): Springer; 2011. p. 145-52.

48. Turner WE. The PushTracker: An activity monitor for manual wheelchair users (student design competition) [Internet]. Arlington (VA): Rehabilitation and Assistive Technology Society of North America; 2011 [cited 2016 Mar 1]. Available from:

http://aac-rerc.psu.edu/wordpressmu/RESNA-SDC/2011/ 04/28/the-pushtracker-an-activity-monitor-for-manualwheelchair-users/

49. Moss AD, Fowler NE, Tolfrey VL. A telemetry-based velocometer to measure wheelchair velocity. J Biomech. 2003;36(2):253-57. [PMID:12547363] http://dx.doi.org/10.1016/S0021-9290(02)00366-4

50. Kiuchi K, Inayama T, Muraoka Y, Ikemoto S, Uemura O, Mizuno K. Preliminary study for the assessment of physical activity using a triaxial accelerometer with a gyro sensor on the upper limbs of subjects with paraplegia driving a wheelchair on a treadmill. Spinal Cord. 2014;52(7):556-63. [PMID:24819509] http://dx.doi.org/10.1038/sc.2014.70

51. Postma K, van den Berg-Emons HJ, Bussmann JB, Sluis TA, Bergen MP, Stam HJ. Validity of the detection of wheelchair propulsion as measured with an Activity Monitor in patients with spinal cord injury. Spinal Cord. 2005; 43(9):550-57. [PMID:15838526] http://dx.doi.org/10.1038/sj.sc.3101759

52. Hiremath SV, Intille SS, Kelleher A, Cooper RA, Ding D. Detection of physical activities using a physical activity monitor system for wheelchair users. Med Eng Phys. 2015; 37(1):68-76. [PMID:25465284] http://dx.doi.org/10.1016/j.medengphy.2014.10.009

53. Bland JM, Altman DG. Statistical methods for assessing agreement between two methods of clinical measurement. Lancet. 1986;1(8476):307-10. [PMID:2868172] http://dx.doi.org/10.1016/S0140-6736(86)90837-8

54. Lahey M, Downey RG, Saal FE. Intraclass correlations: There's more there than meets the eye. Psychol Bull. 1983; 93(3):586-95.

http://dx.doi.org/10.1037/0033-2909.93.3.586

55. Kozey-Keadle S, Libertine A, Lyden K, Staudenmayer J, Freedson PS. Validation of wearable monitors for assessing sedentary behavior. Med Sci Sports Exerc. 2011;43(8): 1561-67. [PMID:21233777] http://dx.doi.org/10.1249/MSS.0b013e31820ce174

56. Arvidsson D. Physical activity and energy expenditure in clinical settings using multisensor activity monitors 
[dissertation]. [Sweden]: Institute of Medicine, Sahlgrenska Academy, University of Gothenburg; 2009.

57. Andre D, Wolf DL. Recent advances in free-living physical activity monitoring: A review. J Diabetes Sci Technol. 2007;1(5):760-67. [PMID:19885145] http://dx.doi.org/10.1177/193229680700100522

58. Steyerberg EW, Vickers AJ, Cook NR, Gerds T, Gonen M, Obuchowski N, Pencina MJ, Kattan MW. Assessing the performance of prediction models: A framework for traditional and novel measures. Epidemiology. 2010;21(1):128-38. [PMID: 20010215] http://dx.doi.org/10.1097/EDE.0b013e3181c30fb2

59. Steyerberg EW, Harrell FE Jr, Borsboom GJ, Eijkemans MJ, Vergouwe Y, Habbema JD. Internal validation of predictive models: Efficiency of some procedures for logistic regression analysis. J Clin Epidemiol. 2001;54(8):774-81. [PMID: 11470385]

60. Bleeker SE, Moll HA, Steyerberg EW, Donders AR, Derksen-Lubsen G, Grobbee DE, Moons KG. External validation is necessary in prediction research: A clinical example. J Clin Epidemiol. 2003;56(9):826-32. [PMID: 14505766]

61. Ambrosio F, Boninger ML, Souza AL, Fitzgerald SG, Koontz AM, Cooper RA. Biomechanics and strength of manual wheelchair users. J Spinal Cord Med. 2005;28(5): 407-14. [PMID:16869087] http://dx.doi.org/10.1080/10790268.2005.11753840

62. Pentland WE, Twomey LT. The weight-bearing upper extremity in women with long term paraplegia. Paraplegia. 1991;29(8):521-30. [PMID:1775358] http://dx.doi.org/10.1038/sc.1991.75

63. Sie IH, Waters RL, Adkins RH, Gellman H. Upper extremity pain in the postrehabilitation spinal cord injured patient. Arch Phys Med Rehabil. 1992;73(1):44-48. [PMID:1729973]
64. Bayley JC, Cochran TP, Sledge CB. The weight-bearing shoulder. The impingement syndrome in paraplegics. J Bone Joint Surg Am. 1987;69(5):676-78. [PMID:3597466]

65. Boninger ML, Dicianno BE, Cooper RA, Towers JD, Koontz AM, Souza AL. Shoulder magnetic resonance imaging abnormalities, wheelchair propulsion, and gender. Arch Phys Med Rehabil. 2003;84(11):1615-20. [PMID:14639560] http://dx.doi.org/10.1053/S0003-9993(03)00282-X

66. Boninger ML, Souza AL, Cooper RA, Fitzgerald SG, Koontz AM, Fay BT. Propulsion patterns and pushrim biomechanics in manual wheelchair propulsion. Arch Phys Med Rehabil. 2002;83(5):718-23. [PMID:11994814] http://dx.doi.org/10.1053/apmr.2002.32455

67. Curtis KA, Black K. Shoulder pain in female wheelchair basketball players. J Orthop Sports Phys Ther. 1999;29(4): 225-31. [PMID:10322595] http://dx.doi.org/10.2519/jospt.1999.29.4.225

Submitted for publication January 13, 2016. Accepted in revised form March 30, 2016.

This article and any supplementary material should be cited as follows:

Tsang K, Hiremath SV, Crytzer TM, Dicianno BE, Ding D. Validity of activity monitors in wheelchair users: A systematic review. J Rehabil Res Dev. 2016;53(6):641-58. http://dx.doi.org/10.1682/JRRD.2016.01.0006

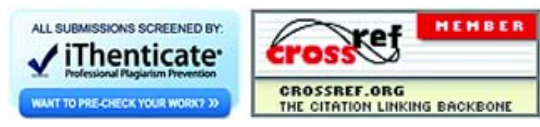


